UDK $577.1: 61$

ISSN 1452-8258

J Med Biochem 39: 363-371, 2020

\title{
IS ENDOCAN A NOVEL POTENTIAL BIOMARKER OF LIVER STEATOSIS AND FIBROSIS?
}

\section{DA LI JE ENDOKAN NOVI POTENCIJALNI BIOMARKER ZA STEATOZU I FIBROZU JETRE?}

\author{
Aleksandra Klisic ${ }^{1}$, Nebojsa Kavaric ${ }^{1}$, Ludovico Abenavoli ${ }^{2}$, Verica Stanisic ${ }^{3}$, \\ Vesna Spasojevic-Kalimanovska ${ }^{4}$, Jelena Kotur-Stevuljevic ${ }^{4}$, Ana Ninic ${ }^{4}$ \\ ${ }^{1}$ Primary Health Care Center, University of Montenegro - Faculty of Medicine, Podgorica, Montenegro \\ ${ }^{2}$ Department of Health Sciences, University Magna Graecia, Catanzaro, Italy \\ ${ }^{3}$ Clinical Center of Montenegro, Podgorica, Montenegro \\ ${ }^{4}$ Department for Medical Biochemistry, University of Belgrade - Faculty of Pharmacy, Belgrade, Serbia
}

\section{Summary}

Background: Studies that evaluated endocan levels in nonalcoholic fatty liver disease (NAFLD) and liver fibrosis are scarce. We aimed to explore endocan levels in relation to different stages of liver diseases, such as NAFLD, as determined with fatty liver index (FLI) and liver fibrosis, as assessed with BARD score.

Methods: A total of 147 participants with $F L I \geq 60$ were compared with 64 participants with FLI <30. An FLI score was calculated using waist circumference, body mass index, gamma-glutamyl transferase and triglycerides. Patients with FLI $\geq 60$ were further divided into those with no/mild fibrosis (BARD score $0-1$ point; $n=23$ ) and advanced fibrosis (BARD score $2-4$ points; $n=124$ ). BARD score was calculated as follows: diabetes mellitus (1 point) + body mass index $\geq 28 \mathrm{~kg} / \mathrm{m}^{2}$ (1 point) + aspartate aminotransferase/alanine aminotransferase ratio $\geq 0.8$ (2 points).

Results: Endocan was independent predictor for FLI and BARD score, both in univariate $[\mathrm{OR}=1.255(95 \% \mathrm{Cl}=$ 1.104-1.426), $\mathrm{P}=0.001 ; \mathrm{OR}=1.208$ (95\% Cl=1.029 1.419), $P=0.021$, respectively] and multivariate binary logistic regression analysis $[\mathrm{OR}=1.287(95 \% \mathrm{Cl}=1.055-$ 1.570), $\mathrm{P}=0.013 ; \mathrm{OR}=1.226(95 \% \mathrm{Cl}=1.022-1.470)$,

\section{Kratak sadržaj}

Uvod: Nema mnogo studija koje su ispitivale vrednosti endokana kod obolelih od nealkoholne steatoze i fibroze jetre. $\mathrm{Na}$ cilj je bio da se ispita nivo endokana u različitim stadijumima oboljenja jetre, kao to su nealkoholna steatoza jetre, predstavljena indeksom masne jetre (FLI) i fibroza jetre, predstavljena BARD skorom.

Metode: Ukupno 147 učesnika sa FLI $\geq 60$ poređeno je sa 64 učesnika sa FLI <30. FLI skor je izračunat koriste i vrednosti obim struka, indeksa telesne mase, aktivnosti gama-glutamil transferaze i vrednosti triglicerida. Ispitanici sa FLI $\geq 60$ su dalje podeljeni u 2 grupe: bez fibroze/blaga fibroza (BARD skor 0-1 poen; $n=23$ ) i uznapredovala fibroza (BARD skor 2-4 poena; $n=124$ ). BARD skor je računat na slede i način: e erna bolest (1 poen) + indeks telesne mase $\geq 28 \mathrm{~kg} / \mathrm{m}^{2}$ (1 poen) + odnos aspartat aminotransferaza/alanin aminotransferaza $\geq 0,8$ ( 2 poena). Rezultati: Endokan je nezavisan prediktor FLI i BARD skora, kako u univarijantnoj $[\mathrm{OR}=1,255(95 \% \mathrm{Cl}=1,104$ 1,426), $\mathrm{P}=0,001$; odnosno $\mathrm{OR}=1,208$ (95\% $\mathrm{Cl}=1,029$ 1,419), $P=0,021]$, tako i u multivarijantnoj binarnoj logističkoj regresionoj analizi $[\mathrm{OR}=1.287(95 \% \mathrm{Cl}=1,055$ 1,570), $P=0,013$; odnosno $O R=1,226(95 \% \mathrm{Cl}=1,022$ 1,470), $P=0,028]$. Endokan kao samostalan prediktor

\footnotetext{
Address for correspondence:

Aleksandra Klisic, MD PhD

Center for Laboratory Diagnostics,

Primary Health Care Center, University of Montenegro

- Faculty of Medicine, Podgorica, Montenegro

Trg Nikole Kovacevica 6, 81000 Podgorica, Montenegro

Phone/Fax: +382 20481999

e-mail: aleksandranklisic@gmail.com
} 
$\mathrm{P}=0.028$, respectively]. Endocan as a single predictor showed poor discriminatory capability for steatosis/fibrosis $[A U C=0.648 ;(95 \% \mathrm{Cl}=0.568-0.727), \mathrm{P}=0.002 ; \mathrm{AUC}=$ 0.667 (95\% Cl=0.555-0.778), $\mathrm{P}=0.013$, respectively], whereas in a Model, endocan showed an excellent clinical accuracy $[A \cup C=0.930 ;(95 \% \mathrm{Cl}=0.886-0.975), \mathrm{P}<0.001$, $A U C=0.840(95 \% \mathrm{Cl}=0.763-0.918), \mathrm{P}<0.001$, respectively].

Conclusions: Endocan independently correlated with both FLI and BARD score. However, when tested in models (with other biomarkers), endocan showed better discriminatory ability for liver steatosis/fibrosis, instead of its usage as a single biomarker.

Keywords: endocan, inflammation, liver steatosis, liver fibrosis, cardiovascular disease

\section{Introduction}

Endocan is a proteoglycan with increased expression in endothelial cells during even the first stage of atheroslerosis (1), which makes this biomarker a convenient parameter of atherosclerosis risk. Not only that it is secreted by endothelial cells, but endocan also stimulates these cells to secrete other inflammation markers (i.e. cytokines), contributes to leukocytes migration, and has an impact on permeability of blood vessels (2-4), all of which further aggravate inflammation and increase cardiovascular disease (CVD) risk burden.

In addition to CVD (1), higher levels of this inflammation biomarker are also reported in states with diminished insulin sensitivity, such as type 2 diabetes $(5,6)$, polycystic ovary syndrome $(7)$, nonalcoholic fatty liver disease (NAFLD) (8), which raises the question whether endocan might represent the link between inflammation in all these mentioned disorders and CVD.

The NAFLD is the most frequent manifestation of hepatic disorders (9). It represents the increased accumulation of lipids which can trigger inflammation (10), and lesions of hepatocytes, that can consequently progress into fibrosis (11). Even more, if not treated fibrosis can further progress into cirrhosis and hepatocellular carcinoma (11).

Increased inflammation and oxidative stress are observed in NAFLD (12-14). These pathophysiological mechanisms can stimulate the synthesis of collagen and induce hepotocytes apoptosis (15). Moreover, the impairment of lipids and lipoprotein concentration was also shown in liver steatosis and fibrosis $(14,16)$, paralell with the increased prevalence of obesity and type 2 diabetes mellitus (17-20).

On the other hand, endocan is not investigated thoroughly in different stages of liver diseases, such as NAFLD and liver fibrosis. Even more, a few studies that examined the concentration of this biomarker in pokazao je slabu diskriminatornu mo za steatozu/fibrozu jetre $[A \cup C=0,648 ;(95 \% \mathrm{Cl}=0,568-0,727), P=0,002$; odnosno $\mathrm{AUC}=0,667(95 \% \mathrm{Cl}=0,555-0,778), \mathrm{P}=0,013]$, ali je u Modelu pokazao odličnu kliničku tačnost [AUC $=0,930 ; \quad(95 \% \mathrm{Cl}=0,886-0,975), \quad \mathrm{P}<0,001 ;$ odnosno $\mathrm{AUC}=0,840(95 \% \mathrm{Cl}=0,763-0,918), \mathrm{P}<0,001]$.

Zaključak: Endokan je nezavisno povezan kako sa FLI, tako i sa BARD skorom. Ipak, u modelu (sa drugim biomarkerima), endokan je pokazao bolju diskriminatornu sposobnost za steatozu/fibrozu jetre.

Ključne reči: endokan, inflamacija, steatoza jetre, fibroza jetre, kardiovaskularne bolesti

fatty liver disease are conflicting (8, 21-23). Since NAFLD represents an independent risk factor for CVD (24), we aimed to explore serum endocan concentration in relation to different stages of liver diseases, such as NAFLD, as determined with fatty liver index (FLI) and liver fibrosis, as assessed with BARD score.

\section{Materials and Methods}

\section{Subjects}

This case-control study included a total of 147 participants with fatty liver diagnosed with $\mathrm{FLI} \geq 60$, who were compared with 64 controls, without fatty liver (i.e., FLI <30). The participants were consecutively recruited when visiting the Primary Health Care Center in Podgorica, Montenegro, for evaluation of metabolic parameters. The survey was conducted during a period from May to July 2017.

The inclusion criteria for participants were NAFLD diagnosed with FLI $\geq 60$ (25) with or without type 2 diabetes. Diabetes was diagnosed by American Diabetes Association Standards of Diabetes Care (26).

Algorithm $\mathrm{FLI}$ is calculated by waist circumference (WC), body mass index (BMI), gamma-glutamyl transferase (GGT) and triglycerides (TG) using equation (25):

$$
\begin{aligned}
& \mathrm{FLI}=\left(\mathrm{e}^{0.953 \times \text { loge }(\mathrm{TG})+0.139 \times \mathrm{BMI}+0.718 \times \text { loge }(\mathrm{GGT})}\right. \\
& +0.053 \times \mathrm{WC}-15.745) /\left(1+\mathrm{e}^{0.953 \times \text { loge }(\mathrm{TG})+0.139 \times \mathrm{BMI}}\right. \\
& +0.718 \times \text { loge }(\mathrm{GGT})+0.053 \times \mathrm{WC}-15.745) \times 100 .
\end{aligned}
$$

An FLI score is regarded to be a reliable algorithm for NAFLD assessment in general population, showing good specificity and sensitivity for NAFLD when diagnosed by abdominal ultrasonography, whereas $\mathrm{FLI} \geq 60$ rules in, and $\mathrm{FLI}<30$ rules out this metabolic disorder $(25,27,28)$. 
Thereafter, only those participants with NAFLD with $\mathrm{FLI} \geq 60$ were further analyzed for potential liver fibrosis. Namely, BARD score (29) was calculated as follows: diabetes mellitus (1 point) $+\mathrm{BMI} \geq 28$ $\mathrm{kg} / \mathrm{m}^{2}$ (1 point) + aspartate aminotransferase (AST)/ alanine aminotransferase (ALT) ratio $\geq 0.8$ (2 points). Harrison et al. (29) showed that a BARD score shows a negative predictive value of $96 \%$ for liver fibrosis.

Accordingly, a total of 147 participants with $F L I \geq 60$ were stratified into two groups: those with no/mild fibrosis (i.e., BARD score 0-1 point; $n=23$ ) and the others with advanced fibrosis (i.e., BARD score 2-4 points; $n=124$ ).

Participants with $30 \leq \mathrm{FLI}<60$, with alcohol consumption ( $>20 \mathrm{~g} /$ day), malignant diseases, type 1 diabetes, with a history of acute myocardial infarction and/or stroke in the last 6 months, renal disease other than diabetic nephropathy, thyroid dysfunction, high sensitivity C-reactive protein (hsCRP) $>10$ $\mathrm{mg} / \mathrm{L}$, and pregnant women were excluded from the examination.

Each participant signed an informed consent and the study protocol was approved by the Ethics Committee of the Primary Health Care Center in Podgorica. The study was performed in conformance with the Declaration of Helsinki ethical guidelines.

Anthropometric parameters (i.e., WC and BMI) were obtained by the same healthcare professional.

The blood pressure was measured after the patient's rest for 5 minutes. The average of the 3 measurements with sphygmomanometer and taken on the right arm was used.

\section{Methods}

The phlebotomy was done between 7: 00 and 10:00 a.m. after an over night fast of at least 8 hours. Blood samples were taken in tubes with serum separator and clot activator, and each sample was left to clot within half an hour. Afterwards, the samples were centrifuged at $3000 \mathrm{xg}$, at room temperature for 10 minutes. Sera were divided into aliquots and stored at $-80{ }^{\circ} \mathrm{C}$ for determination of endocan and hsCRP, whereas one aliquot of each serum was analysed immediately after centrifugation for lipid parameters [i.e., total cholesterol (TC), TG, low density lipoprotein cholesterol (LDL-c), and high density lipoprotein cholesterol (HDL-c)], glucose, AST, ALT, GGT and creatinine. All these parameters were determined on Roche Cobas c501 chemistry analyzer (Roche Diagnostics GmbH, Mannheim, Germany). Serum endocan level was determined by using an enzyme-linked immunosorbent commercial assay (ab213776 - Human ESM1 ELISA Kit, Abcam, Cambridge, UK), whereas serum hsCRP levels were determined nephelometrically (Behring Nephelometer Analyzer, Marburg, Germany).

\section{Statistical analysis}

Statistical analysis was done using SPSS 21.0 (SPSS Inc., Chicago, USA). Data distribution was tested by Shapiro Wilk test. Continuous variables were presented as median (interquartile range) and tested by Mann-Whitney test. Categorical variables were presented as absolute and relative frequencies and analyzed using the Chi-square test for contingency tables. Spearman's correlation analysis ( $\rho$ ) was applied to examine the associations between FLI and BARD score with the clinical data. Furthermore, binary logistic regression analysis was applied in order to test the independent associations of endocan with FLI and BARD score. When performing logistic regression analysis, $\mathrm{FLI}<30$ was used as a reference and $F L I \geq 60$ as risk categories, while BARD scores 0 and 1 point were used as reference (i.e., no/mild fibrosis) and BARD scores 2, 3 and 4 points were used as risk categories (i.e., advanced fibrosis). Multivariate logistic regression analysis was used to investigate possible independent predictions of endocan on the presence of fatty liver, as well as on advanced fibrosis. Data from binary logistic regression analysis were showed as odds ratio (OR) and 95\% confidence interval $(\mathrm{Cl})$. Receiver operating characteristic $(\mathrm{ROC})$ analysis and the area under the ROC curve $(A \cup C)$ were used to test the predictive ability of endocan, solely and in a model, for identifying patients with fatty liver and advanced fibrosis. Clinical accuracy of endocan was analysed according to Swets (30). The P value less than 0.05 was considered as statistically significant.

\section{Results}

Clinical data of participants are summarized in Table I. Significantly more men were found in the group with fatty liver than in the group without it. Individuals with fatty liver displayed greater BMI, WC, as well as higher prevalence of type 2 diabetes, as compared with individuals without fatty liver. More subjects with fatty liver used antihyperglycemic, insulin and antihypertensive therapy, than those without fatty liver. Also, they had significantly higher glucose, HbA1c, TG, ALT, GGT, hsCRP and endocan levels, but lower HDL-c levels than individuals without fatty liver.

The correlation coefficients from Spearman's correlation analysis between $\mathrm{FLI}$ and clinical data were showed in Table II. BMI, WC, glucose, HbA1c, TG, ALT, GGT and hsCRP showed positive, but HDL$c$ negative correlations with FLI.

Binary logistic regression analysis was applied to determine in-depth associations of endocan and the presence of fatty liver disease. In univariate analysis, endocan showed significant relation with fatty liver disease $[O R=1.255 \quad(95 \% \quad \mathrm{Cl}=1.104-1.426)$, $P=0.001]$. Negelkerke $R^{2}$ in univariate regression 
Table I Clinical data and endocan levels in patients without and with fatty liver.

\begin{tabular}{|c|c|c|c|}
\hline & Without fatty liver (FLI <30) & Fatty liver (FLI $\geq 60)$ & $P$ \\
\hline $\mathrm{N}$ (male, \%) & $64(14 \%)$ & 147 (47\%) & $<0.001$ \\
\hline Age, years & $60(52-65)$ & $62(57-68)$ & 0.051 \\
\hline $\mathrm{BMI}, \mathrm{kg} / \mathrm{m}^{2}$ & $24.1(23.1-25.3)$ & $31.6(29.9-33.8)$ & $<0.001$ \\
\hline $\mathrm{WC}, \mathrm{cm}$ & $85(81-89)$ & $105(100-111)$ & $<0.001$ \\
\hline $\mathrm{SBP}, \mathrm{mmHg}$ & $136(126-151)$ & $130(125-145)$ & 0.243 \\
\hline $\mathrm{DBP}, \mathrm{mmHg}$ & $86(77-94)$ & $83(76-92)$ & 0.345 \\
\hline Diabetes, n (\%) & $10(16 \%)$ & $75(51 \%)$ & $<0.001$ \\
\hline Smokers, n (\%) & $15(23 \%)$ & $23(16 \%)$ & 0.176 \\
\hline Antihyperglycemics, n (\%) & $7(11 \%)$ & $65(44 \%)$ & $<0.001$ \\
\hline Insulin therapy, n (\%) & $2(3 \%)$ & $22(15 \%)$ & 0.016 \\
\hline Antihypertensives, n (\%) & $31(48 \%)$ & $111(76 \%)$ & $<0.001$ \\
\hline Hypolipidemics, n (\%) & $18(28 \%)$ & $58(39 \%)$ & 0.122 \\
\hline FLI & $16(13-22)$ & $83(70-91)$ & $<0.001$ \\
\hline Glucose, $\mathrm{mmol} / \mathrm{L}$ & $5.5(5.3-5.8)$ & $6.4(5.5-8.3)$ & $<0.001$ \\
\hline HbA1c, \% & $5.4(5.2-5.7)$ & $6.0(5.5-7.2)$ & $<0.001$ \\
\hline $\mathrm{HbA} 1 \mathrm{c}, \mathrm{mmol} / \mathrm{mol}$ & $36(33-39)$ & $42(37-55)$ & $<0.001$ \\
\hline $\mathrm{TC}, \mathrm{mmol} / \mathrm{L}$ & $5.75(4.75-6.54)$ & $5.83(4.87-6.93)$ & 0.475 \\
\hline HDL-cholesterol, mmol/L & $1.73(1.53-2.03)$ & $1.19(1.00-1.40)$ & $<0.001$ \\
\hline LDL-cholesterol, mmol/L & $3.10(2.52-4.025)$ & $3.45(2.62-4.53)$ & 0.254 \\
\hline $\mathrm{TG}, \mathrm{mmol} / \mathrm{L}$ & $1.17(0.94-1.43)$ & $2.25(1.71-2.95)$ & $<0.001$ \\
\hline AST, U/L & $20(17-23)$ & $20(17-24)$ & 0.646 \\
\hline $\mathrm{ALT}, \mathrm{U} / \mathrm{L}$ & $17(11-22)$ & $22(17-32)$ & $<0.001$ \\
\hline GGT, U/L & $12(9-16)$ & $22(16-31)$ & $<0.001$ \\
\hline $\mathrm{HsCRP}, \mathrm{mg} / \mathrm{L}$ & $0.52(0.32-0.98)$ & $1.70(0.77-3.11)$ & $<0.001$ \\
\hline Endocan, ng/L & $27.8(17.6-40.9)$ & $38.8(21.6-89.5)$ & 0.002 \\
\hline
\end{tabular}

Data are given as median (interquartile range) and compared by Mann-Whitney test.

BMI - Body mass index; WC - Waist circumference; SBP - Systolic blood pressure; DBP - Diastolic blood pressure; FLI - Fatty liver index; HbA1c - Glycated hemoglobin; TC - Total cholesterol; HDL-cholesterol - High density lipoprotein cholesterol; LDLcholesterol - Low density lipoprotein cholesterol; TG - Triglycerides; AST - Aspartate aminotransferase; ALT - Alanine aminotransferase; GGT - Gamma-glutamyl transferase; HsCRP - High-sensitivity C-reactive protein

Table II Spearman's correlation analysis of clinical markers and FLI.

\begin{tabular}{|l|c|c|}
\hline Variable & $\rho$ & $P$ \\
\hline Age, years & 0.121 & 0.079 \\
\hline BMl, $\mathrm{kg} / \mathrm{m}^{2}$ & 0.840 & $<0.001$ \\
\hline WC, cm & 0.902 & $<0.001$ \\
\hline SBP, $\mathrm{mmHg}$ & -0.038 & 0.578 \\
\hline DBP, $\mathrm{mmHg}$ & -0.052 & 0.456 \\
\hline Glucose, mmol/L & 0.435 & $<0.001$ \\
\hline $\mathrm{HbA1c} / \%$ & 0.397 & $<0.001$ \\
\hline TC, mmol/L & -0.026 & 0.709 \\
\hline $\mathrm{HDL}-\mathrm{cholesterol,} \mathrm{mmol/L}$ & -0.504 & $<0.001$ \\
\hline LDL-cholesterol, mmol/L & -0.028 & 0.685 \\
\hline TG, mmol/L & 0.582 & $<0.001$ \\
\hline AST, U/L & 0.109 & 0.115 \\
\hline ALT, U/L & 0.390 & $<0.001$ \\
\hline GGT, U/L & 0.639 & $<0.001$ \\
\hline HsCRP, mg/L & 0.413 & $<0.001$ \\
\hline Endocan, ng/L & 0.203 & 0.005 \\
\hline
\end{tabular}

Data age given as coefficients of correlation Rho $(\rho)$.

BMI-Body mass index; WC-Waist circumference; SBP-Systolic blood pressure; DBP-Diastolic blood pressure; HbA1c-Glycated hemoglobin; TC-Total cholesterol; HDL-cholesterol-High density lipoprotein cholesterol; LDL-cholesterol-Low density lipoprotein cholesterol; TG-Triglycerides; AST-Aspartate aminotransferase; ALT-Alanine aminotransferase; GGT-Gammaglutamyl transferase; HsCRP-High-sensitivity C-reactive protein 


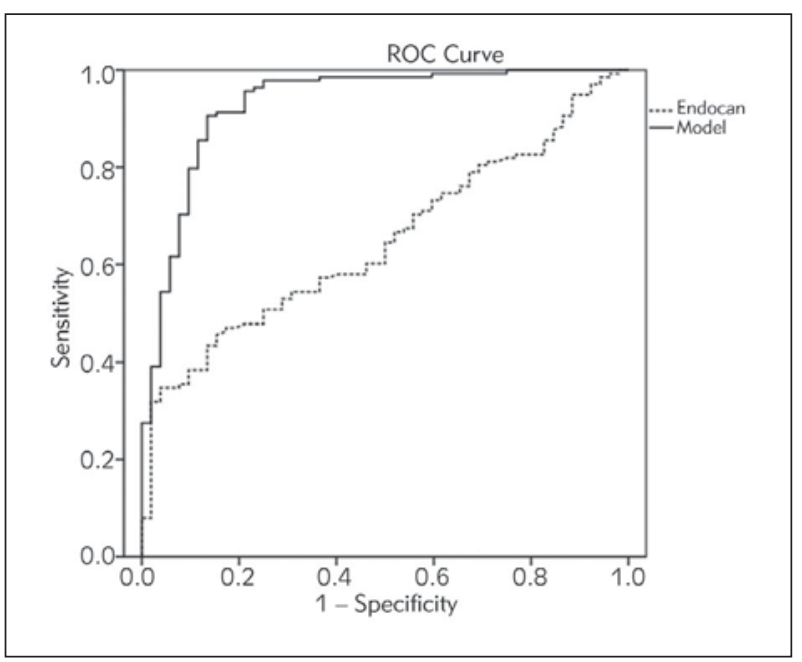

Figure 1 ROC curves of endocan and selected Model discriminatory capabilities towards presence of fatty liver. analysis was 0.125. Markers significantly correlated with FLI in non-parametric Spearman's correlation analysis (i.e., HbA1c, HDL-c, ALT, hsCRP and endocan), but not used for FLI calculation, as well as demographic characteristics significantly different between FLI groups (i.e., gender, antihyperglycemic, insulin and antihypertensive therapies) were used in Model to test the independent prediction of endocan for fatty liver disease. Endocan was shown to be an independent predictor for fatty liver $[\mathrm{OR}=1.287$ (95\% Cl=1.055-1.570), $P=0.013]$. Nagelkerke $R^{2}$ of 0.656 demonstrated that even $65.6 \%$ of variation in fatty liver disease occurrence could be explained by this Model.

ROC analysis was used to discriminate patients with fatty liver from those without fatty liver (Figure 1). Endocan as a single predictor showed poor discrimi-

Table III Clinical data and endocan levels in patients without and with advanced fibrosis.

\begin{tabular}{|c|c|c|c|}
\hline & No/mild fibrosis & Advanced fibrosis & $\mathrm{P}$ \\
\hline $\mathrm{N}$ (male, \%) & $23(70 \%)$ & $124(43 \%)$ & 0.018 \\
\hline Age, years & $56(51-61)$ & $64(58-70)$ & $<0.001$ \\
\hline $\mathrm{BMI}, \mathrm{kg} / \mathrm{m}^{2}$ & $29.7(28.1-33.3)$ & $31.8(30.3-34.3)$ & 0.020 \\
\hline WC, $\mathrm{cm}$ & 105 (99-107) & 107 (101-112) & 0.233 \\
\hline $\mathrm{SBP}, \mathrm{mmHg}$ & $139(126-146)$ & $130(125-144)$ & 0.586 \\
\hline $\mathrm{DBP}, \mathrm{mmHg}$ & $84(80-92)$ & $83(76-90)$ & 0.314 \\
\hline Diabetes, n (\%) & $4(17 \%)$ & $71(57 \%)$ & $<0.001$ \\
\hline Smokers, n (\%) & $5(22 \%)$ & $18(15 \%)$ & 0.381 \\
\hline Antihyperglycemics, n (\%) & $3(13 \%)$ & $62(50 \%)$ & 0.001 \\
\hline Insulin, n (\%) & $3(13 \%)$ & $19(15 \%)$ & 0.778 \\
\hline Antihypertensives, n (\%) & $12(52 \%)$ & $99(80 \%)$ & 0.005 \\
\hline Hypolipidemics, n (\%) & $8(36 \%)$ & $50(40 \%)$ & 0.618 \\
\hline Glucose, $\mathrm{mmol} / \mathrm{L}$ & $5.4(5.2-6.1)$ & $6.7(5.8-8.5)$ & $<0.001$ \\
\hline $\mathrm{HbA} 1 \mathrm{c}, \%$ & $5.5(5.3-5.9)$ & $6.3(5.6-7.3)$ & 0.002 \\
\hline $\mathrm{HbA} 1 \mathrm{c}, \mathrm{mmol} / \mathrm{mol}$ & $37(34-41)$ & $45(37-56)$ & 0.002 \\
\hline $\mathrm{TC}, \mathrm{mmol} / \mathrm{L}$ & $6.59(5.04-7.79)$ & $5.78(4.87-5.79)$ & 0.209 \\
\hline HDL-cholesterol, mmol/L & $1.21(1.01-1.32)$ & $1.19(0.99-1.34)$ & 0.841 \\
\hline LDL-cholesterol, mmol/L & $3.94(3.07-4.86)$ & $3.40(2.60-4.37)$ & 0.151 \\
\hline $\mathrm{TG}, \mathrm{mmol} / \mathrm{L}$ & $2.36(1.73-2.84)$ & $2.20(1.70-2.97)$ & 0.821 \\
\hline AST, U/L & $20(19-24)$ & $20(17-25)$ & 0.443 \\
\hline $\mathrm{ALT}, \mathrm{U} / \mathrm{L}$ & $31(25-39)$ & $21(16-28)$ & $<0.001$ \\
\hline GGT, U/L & $23(16-35)$ & $22(15-30)$ & 0.552 \\
\hline $\mathrm{HsCRP}, \mathrm{mg} / \mathrm{L}$ & $1.81(0.46-2.94)$ & $1.67(0.80-3.11)$ & 0.362 \\
\hline Endocan, ng/L & $26.0(14.2-44.3)$ & $44.2(22.8-92.7)$ & 0.013 \\
\hline
\end{tabular}

Data are given as median (interquartile range) and compared by Mann-Whitney test. 
Table IV Spearman's correlation analysis of clinical markers and BARD.

\begin{tabular}{|l|c|c|}
\hline Variable & $\rho$ & P \\
\hline Age, years & 0.419 & 0.080 \\
\hline BMI, $\mathrm{kg} / \mathrm{m}^{2}$ & 0.145 & 0.529 \\
\hline $\mathrm{WC}, \mathrm{cm}$ & 0.052 & 0.782 \\
\hline $\mathrm{SBP}, \mathrm{mmHg}$ & 0.023 & $<.842$ \\
\hline DBP, $\mathrm{mmHg}$ & 0.017 & $<0.001$ \\
\hline Glucose, $\mathrm{mmol} / \mathrm{L}$ & 0.349 & $<0.001$ \\
\hline $\mathrm{HbA} 1 \mathrm{c}, \%$ & 0.307 & 0.085 \\
\hline TC, mmol/L & -0.142 & 0.074 \\
\hline $\mathrm{HDL}-\mathrm{cholesterol}, \mathrm{mmol} / \mathrm{L}$ & 0.148 & 0.086 \\
\hline LDL-cholesterol, $\mathrm{mmol} / \mathrm{L}$ & -0.142 & 0.018 \\
\hline TG, mmol/L & -0.195 & 0.522 \\
\hline AST, U/L & -0.049 & $<0.001$ \\
\hline ALT, U/L & -0.461 & 0.110 \\
\hline GGT, U/L & -0.131 & 0.457 \\
\hline HsCRP, mg/L & 0.062 & 0.010 \\
\hline Endocan, $\mathrm{ng} / \mathrm{L}$ & 0.217 & \\
\hline
\end{tabular}

Data age given as coefficients of correlation Rho $(\rho)$.

BMI - Body mass index; WC - Waist circumference; SBP - Systolic blood pressure; DBP - Diastolic blood pressure; HbA1c Glycated hemoglobin; TC - Total cholesterol; HDL-cholesterol - High density lipoprotein cholesterol; LDL-cholesterol - Low density lipoprotein cholesterol; TG - Triglycerides; AST - Aspartate aminotransferase; ALT - Alanine aminotransferase; GGT Gamma-glutamyl transferase; HsCRP - High-sensitivity C-reactive protein

natory capability $[\mathrm{AUC}=0.648 ; \quad(95 \% \quad \mathrm{Cl}=0.568$ $0.727), P=0.002]$. Specificity of this test was $96.16 \%$, but sensitivity was $34.78 \%$. On the contrary, when tested in the Model, endocan showed an excellent clinical accuracy $[\mathrm{AUC}=0.930 ;(95 \% \mathrm{Cl}=0.886$ $0.975)]$ with sensitivity of $90.58 \%$ and specificity of $86.54 \%$.

Only patients with fatty liver ( $\mathrm{FLI} \geq 60$ ) were further tested for possible presence of advanced fibrosis. Their characteristics were given in Table III. Significantly more women were found among those with advanced than with no/mild fibrosis. Patients with advanced fibrosis were older, had higher BMI, and used frequently more antihyperglycemic and antihypertensive therapies than those with no/mild fibrosis. Also, significantly more of them had diabetes. Glucose, HbA1c, ALT and endocan levels were higher in those with advanced fibrosis than in individuals with no/mild fibrosis.

Years of age, glucose, $\mathrm{HbA} 1 \mathrm{c}$, and endocan correlated significantly positively with BARD score (Table IV). On the contrary, ALT and TG correlated significantly negatively with BARD score.

Binary logistic regression analysis was used to test the associations of endocan with the presence of advanced fibrosis. In univariate analysis odds ratio for endocan was $\mathrm{OR}=1.208(95 \% \mathrm{Cl}=1.029-1.419)$, $\mathrm{P}=0.021, \mathrm{R}^{2}=0.088$. In multivariate analysis, in the Model that was consisted of gender, ages, TG, antihypertensive therapy and endocan, the latest was shown

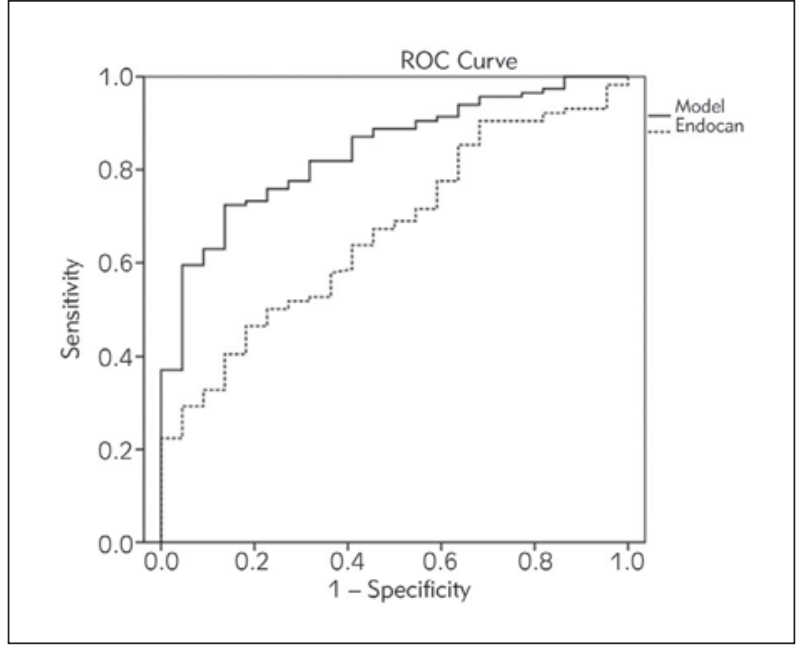

Figure 2 ROC curves of endocan and selected Model discriminatory capabilities towards presence of advanced fibrosis.

to be the independent predictor of advanced fibrosis $[\mathrm{OR}=1.226$ (95\% Cl=1.022-1.470), $\mathrm{P}=0.028]$. Clinical markers that entered the Model were continuous variables which showed significant correlation with BARD score in Spearman's correlation analysis (Table IV) and categorical data significantly different between no/mild vs. advanced fibrosis (Table III). Nagelkerke $\mathrm{R}^{2}$ for the Model was 0.330 which shows that $33 \%$ of variation in the presence of advanced fibrosis could be explained by this Model. 
ROC analysis showed that curve for the Model (Figure 2) had an excellent discriminatory capability $[A U C=0.840 \quad(95 \% \quad C l=0.763-0.918), \quad P<0.001]$ towards advanced fibrosis with specificity of $86.36 \%$ and sensitivity of $72.41 \%$. On the other hand, endocan itself has poor discriminatory ability towards advanced fibrosis [AUC $=0.667 \quad(95 \% \mathrm{Cl}=0.555-$ $0.778), P=0.013]$ with specificity $81.82 \%$ and sensitivity $46.55 \%$.

\section{Discussion}

Our study shows that endocan levels were higher in NAFLD (as assessed with FLI), as well as in advanced fibrosis (as assessed with BARD score), as compared with controls. Importantly, endocan independently correlated with both, FLI and BARD score.

Only a small number of previous studies examined this biomarker in NAFLD, and showed contradictory results $(8,21-23)$. Tok et al. (22) showed lower endocan concentration in 38 patients with NAFLD, whereas Dallilo et al. (8) reported vice versa (i.e., its higher levels in 19 patients with NAFLD and 32 with type 2 diabetes mellitus with NAFLD), as compared with controls. On the contrary, Ustyol et al. (23) found no difference in serum endocan levels between obese participants with and without NAFLD, as compared with controls in pediatric population.

Also, previous study (31) investigated serum endocan in patients with liver cirrhosis and found only higher level in patients with decompensated cirrhosis, but no difference between compensated cirhhosis and healthy controls was observed.

Additionally, another study showed that patients with NAFLD and coronary artery disease displayed higher serum endocan levels as compared with patients with NAFLD, but without coronary artery disease (21).

Possible discrepancies in those results might be explained by the fact that sample size of participants differed between the studies. In our study we included a relatively larger sample size than previous studies did (i.e., a total of 147 participants with NAFLD), and only adult Caucasians (i.e., Montenegrin) population $(8,22,23)$.

Endocan is highly expressed in endothelial cell injury (1) with its up-regulation observed in the presence of proangiogenic molecules and proinflammatory cytokines (2). The pathological accumulation of lipids in NAFLD triggers inflammation (10), which can lead to hepatocytes dysfunction. Additionally, paralell with the liver fibrosis and with the processes of its repairment, increased production of extracellular matrix proteins occurs (11). Since proteoglycans are constitute of the extracellular matrix, acting as its structural components (32), this might explain the higher level of endocan, as one of the proteoglycans, in liver fibrosis.

Considering the fact that NAFLD is an independent predictor for CVD (24), not only that endocan might reflect the severity of liver failure, but further prospective studies are necessary to explore the causal link between high endocan level, liver steatosis/fibrosis and CVD.

In our study median endocan level in patients with NAFLD was $38.8 \mathrm{ng} / \mathrm{L}$ (21.6-89.5), whereas in advanced fibrosis was $44.2 \mathrm{ng} / \mathrm{L}$ (22.8-92.7), as compared with non-NAFLD group [median 27.8 $\mathrm{ng} / \mathrm{L}$ (17.6-40.9)], thus presuming its increase with progression of liver disease.

Although an independent association between liver steatosis/fibrosis and serum endocan level is shown in the current study, this proteoglycan seems to be more convenient in the diagnostic evaluation of these liver disorders, in combination with other markers, instead of its usage alone, as a single biomarker. Namely, in our study endocan showed poor discriminatory capability $(A \cup C=0.648)$ for NAFLD as a single predictor. On the contrary, when tested in the Model [i.e., variables that entered the Model were: gender, $\mathrm{HbA} 1 \mathrm{c}$, insulin, antihyperglycemic and antihypertensive therapies (categorical variables), and HDL-c, ALT, hsCRP, endocan (continuous variables)], endocan showed an excellent clinical accuracy ( $A \cup C=0.930$ ) with sensitivity of $90.58 \%$ and specificity of $86.54 \%$.

We obtained the similar results when evaluating the diagnostic accuracy of endocan in liver fibrosis. Namely, endocan itself showed poor discriminatory ability for advanced fibrosis (AUC=0.667). However, when tested in the Model [i.e., variables that entered the Model: gender, antihypertensive therapy (categorical variables) and ages, TG, endocan (continuous variables)], endocan showed an excellent discriminatory capability ( $A \cup C=0.840$ ) for advanced fibrosis, with specificity of $86.36 \%$ and sensitivity of $72.41 \%$.

Our previous studies have also demonstrated the benefits of multimarker approach in better discrimination of individuals with liver steatosis (12-14). Namely, an independent relationship between FLI and insulin resistance (i.e. HOMA-IR) and inflammation (i.e. hsCRP) was recorded in the cohort of postmenopausal women (13). Also, ALT was shown to be independently correlated with $\mathrm{FLI}$ in both genders (12) in a large Montenegrin population sample, whereas HDL-c and malondialdehyde independently correlated with FLI in the cohort of patients with type 2 diabetes mellitus (14). However, when tested in model with other lipid, inflammation and oxidative stress biomarkers, the discriminative ability for liver steatosis development was significantly enhanced (14). These mentioned results point out that in addition to traditional risk factors, multimarker approach 
including cluster of different biomarkers may significantly improve the timely identification of those patients with high risk of liver steatosis. Moreover, in another study we have also reported that older age and higher HDL-C are independently correlated with advanced liver fibrosis assessed with the BARD score, suggesting that further examination of enzymes involved in lipoprotein metabolism could be perspective for revealing the causal association between lipid parameters and liver fibrosis (16).

The main disadvantage of this study is its crosssectional design which limits us to conclude the cause-effect between high endocan and liver steatosis/fibrosis. Additionally, we were not able to use imaging diagnostic procedures, but simple and easy obtained algorithms, like previous studies did $(28,33)$. However, the 2016 European Association for the Study of the Liver (EASL), European Association for the Study of Diabetes (EASD), and European Association for the Study of Obesity (EASO), recommended the usage of the $\mathrm{FLI}$ as one of the best validated steatosis scores for screening studies in large samples (28). Additionally, BARD score is shown to be useful for ruling out advanced fibrosis, thus reducing the need for liver biopsies in NAFLD patients $(34,35)$.

\section{References}

1. Balta S, Mikhailidis DP, Demirkol S, Ozturk C, Celik T, lyisoy $A$. Endocan: A novel inflammatory indicator in cardiovascular disease? Atherosclerosis 2015; 243(1): 339-43.

2. Zhao T, Kecheng Y, Zhao X, Hu X, Zhu J, Wang Y, et al. The higher serum endocan levels may be a risk factor for the onset of cardiovascular disease: A meta-analysis. Medicine (Baltimore) 2018; 97(49): e13407.

3. Qiu CR, Fu Q, Sui J, Zhang Q, Wei P, Wu Y, et al. Analysis of Serum Endothelial Cell-Specific Molecule 1 (Endocan) Level in Type 2 Diabetes Mellitus With Acute ST-Segment Elevation Myocardial Infarction and its Correlation: A Pilot Study. Angiology 2017; 68(1): 74-8.

4. Lee W, Ku SK, Kim SW, Bae JS. Endocan elicits severe vascular inflammatory responses in vitro and in vivo. J Cell Physiol 2014; 229: 620-30.

5. Ekiz-Bilir B, Bilir B, Aydın M, Soysal-Atile N. Evaluation of endocan and endoglin levels in chronic kidney disease due to diabetes mellitus. Arch Med Sci 2019; 15(1): 8691.

6. Klisic A, Kavaric N, Stanisic V, Vujcic S, SpasojevicKalimanovska V, Ninic A, et al. Endocan and a novel score for dyslipidemia, oxidative stress and inflammation (DOI score) are independently correlated with glycated hemoglobin (HbA1c) in patients with prediabetes and type 2 diabetes. Arch Med Sci 2019; doi: https: //doi.org/10.5114/aoms.2019.87541.

\section{Conclusion}

Higher serum endocan levels were observed in NAFLD (as determined with FLI) and in advanced fibrosis (as determined with BARD score), as compared with controls. Importantly, endocan was independently correlated with both, FLI and BARD score. However, when tested in models (with other biomarkers), this proteoglycan showed better discriminatory ability for liver steatosis/fibrosis, instead of its usage alone, as a single biomarker.

Acknowledgement. This work was financially supported in part by a grant from the Ministry of Science, Montenegro and the Ministry of Education, Science and Technological Development, Republic of Serbia (project number 175035).

\section{Conflict of interest statement}

The authors state that they have no conflicts of interest regarding the publication of this article.

7. Bicer M, Guler A, Unal Kocabas G, Imamoglu C, Baloglu $A$, Bilgir $O$, et al. Endocan is a predictor of increased cardiovascular risk in women with polycystic ovary syndrome. Endocr Res 2017; 42(2): 145-53.

8. Dallio M, Masarone M, Caprio GG, Di Sarno R, Tuccillo C, Sasso FC, et al. Endocan Serum Levels in Patients with Non-Alcoholic Fatty Liver Disease with or without Type 2 Diabetes Mellitus: A Pilot Study. J Gastrointestin Liver Dis 2017; 26(3): 261-8.

9. Abenavoli L, Pellicano R, Boccuto L. Role of genetics and metabolism in non-alcoholic fatty liver disease. Panminerva Med 2018; 60(2): 41-3.

10. Yu E, Hsu HY, Huang CY, Hwang LC. Inflammatory Biomarkers and Risk of Atherosclerotic Cardiovascular Disease. Open Med (Wars) 2018;13: 208-13.

11. Abenavoli L, Milic N, Di Renzo L, Preveden T, MedićStojanoska M, De Lorenzo A. Metabolic aspects of adult patients with nonalcoholic fatty liver disease. World J Gastroenterol 2016; 22(31): 7006-16.

12. Klisic A, Kavaric N, Ninic A. Predictive values of serum uric acid and alanine-aminotransferase for fatty liver index (FLI) in Montenegrin population. J Med Biochem 2019; 38(4): 407-17.

13. Klisic A, Kavaric N, Jovanovic M, Soldatovic I, GligorovicBarhanovic N, Kotur-Stevuljevic J. Bioavailable testosterone is independently associated with fatty liver index in 
postmenopausal women. Arch Med Sci 2017; 5(13): $1188-96$.

14. Klisic A, Isakovic A, Kocic G, Kavaric N, Jovanovic $M$, Zvrko $E$, et al. Relationship between Oxidative Stress, Inflammation and Dyslipidemia with Fatty Liver Index in Patients with Type 2 Diabetes Mellitus. Exp Clin Endocrinol Diabetes 2018; 126(06): 371-8.

15. Begriche K, Massart J, Robin MA, Bonnet F, Fromenty B. Mitochondrial adaptations and dysfunctions in nonalcoholic fatty liver disease. Hepatology 2013; 58: 14971507.

16. Klisic A, Abenavoli L, Fagoonee S, Kavaric N, Kocic G, Ninić A. Older age and HDL-cholesterol as independent predictors of liver fibrosis assessed by BARD score. Minerva Med 2019; 110(3): 191-8.

17. Sayın S, Kutlu R, Kulaksızoğlu M. The relationship between sex steroids, insulin resistance and body compositions in obese women: A case-control study. J Med Biochem 2020: 39: 25-31.

18. Maksimovic $M$, Vlajinac $H$, Radak $D j$, Marinkovic J, Maksimovic J, Jorga J. Association of overweight and obesity with cardiovascular risk factors in patients with atherosclerotic diseases. J Med Biochem 2020; 39: 215-23.

19. Naghizadeh $M$, Saghafi-Asl M, Amiri P, Karamzad N. Lipid profile in relation to inflammatory and insulin resistance markers and anthropometric indices in the apparently healthy abdominally obese. Prog Nutr 2019; 21(1-S): 370-7.

20. Moradi M, Alvandi E, Koohdani F. The Effect of obesity and weight loss through calorie restriction on HDL function. Prog Nutr 2019; 21(1-S): 16-24.

21. Elsheikh E, Younoszai Z, Otgonsuren M, Hunt $S$, Raybuck B, Younossi ZM. Markers of endothelial dysfunction in patients with non-alcoholic fatty liver disease and coronary artery disease. J Gastroenterol Hepatol 2014; 29(7): 1528-34.

22. Tok D, Ekiz F, Basar O, Coban S, Ozturk G. Serum endocan levels in patients with chronic liver disease. Int J Clin Exp Med 2014; 7(7): 1802-7.

23. Ustyol A, Aycan Ustyol E, Gurdol F, Kokali F, Bekpınar S. Pselectin, endocan, and some adhesion molecules in obese children and adolescents with non-alcoholic fatty liver disease. Scand J Clin Lab Invest 2017; 77(3): 205-9.

24. Wu S, Wu F, Ding Y, Hou J, Bi J, Zhang Z. Association of non-alcoholic fatty liver disease with major adverse cardiovascular events: A systematic review and meta-analysis. Sci Rep 2016; 6: 33386.
25. Bedogni G, Bellentani S, Miglioli L, Masutti F, Passalacqua $M$, Castiglione $A$, et al. The fatty liver index: a simple and accurate predictor of hepatic steatosis in the general population. BMC Gastroenterol 2006; 6: 44.

26. American Diabetes Association. Classification and diagnosis of diabetes. Sec. 2. In Standards of Medical Care in Diabetes-2017. Diabetes Care 2017; 40: S11-S24.

27. Zelber Sagi S, Webb M, Assy N, Blendis L, Yeshua $H$, Leshno $M$, et al. Comparison of fatty liver index with noninvasive methods for steatosis detection and quantification. World J Gastroenterol 2013; 19(1): 57-64.

28. European Association for the Study of the Liver (EASL). Electronic address: easloffice@easloffice.eu, European Association for the Study of Diabetes (EASD), European Association for the Study of Obesity (EASO). EASLEASD-EASO Clinical Practice Guidelines for the management of non-alcoholic fatty liver disease. J Hepatol 2016; 64: 1388-402.

29. Harrison SA, Oliver D, Arnold HL, Gogia S, Neuschwander-Tetri BA. Development and validation of a simple NAFLD clinical scoring system for identifying patients without advanced disease. Gut 2008; 57: 1441-7.

30. Swets JA. Measuring the accuracy of diagnostic systems. Science 1988; 240: 1285-93.

31. Zuwala-Jagiello J, Simon K, Kukla M, MurawskaCialowicz E, Gorka-Dynysiewicz J, Grzebyk E, et al. Increased circulating endocan in patients with cirrhosis: relation to bacterial infection and severity of disease. J Physiol Pharmacol 2017; 68(2): 273-82.

32. Baghy K, Tátrai P, Reg s E, Kovalszky I. Proteoglycans in liver cancer. World J Gastroenterol 2016; 22(1): 379-93.

33. Neukam K, Bhagani S, Roger A, Oben J, Nirmal D, Jain $A$, et al. High prevalence of non-alcoholic fatty liver disease (NAFLD) among Gujarati Indians in North London: a population based study. Clinical Lipidology 2017; 12: 33-9.

34. Raszeja-Wyszomirska J, Szymanik B, Ławniczak M, Kajor M, Chwist A, Milkiewicz P, et al. Validation of the BARD scoring system in Polish patients with nonalcoholic fatty liver disease (NAFLD). BMC Gastroenterol 2010; 10: 67.

35. Cicho-Lach H, Celi ski K, Prozorow-Król B, Swatek J, Słomka M, Lach T. The BARD score and the NAFLD fibrosis score in the assessment of advanced liver fibrosis in nonalcoholic fatty liver disease. Med Sci Monit 2012; 18(12): 735-40. 